\title{
Electrosurgery and Implantable Electronic Devices: Review and Implications for Office-Based Procedures
}

\author{
Melissa A. Voutsalath, DO ${ }^{*}$ Christopher K. Bichakjian, MD, ${ }^{*}$ Frank Pelosi, MD ${ }^{\dagger}$ \\ David Blum, MD ${ }^{\ddagger}$ Timothy M. Johnson, MD ${ }^{* \S}$ and Peter M. Farrehi, $\mathrm{MD}^{\dagger}$
}

The authors have indicated no significant interest with commercial supporters.

$\mathrm{T}$ he number of patients with implantable electronic devices (IEDs) is growing as indications for a myriad of cardiac and neurological disorders that cannot be managed using medication alone expand. ${ }^{1}$ These devices include gastric and cardiac pacemakers, implantable cardioverter defibrillators, cochlear implants, and a range of stimulators of the deep brain, vagal nerve, sacral nerve, phrenic nerve, spinal cord, and bone. Encountering patients with an IED who present for office-based procedures is becoming increasingly common. Electromagnetic interference (EMI) during routine procedures has been reported to be associated with inappropriate functioning of IEDs. ${ }^{2}$ Adverse effects of performing electrosurgery on patients with IEDs pose a patient safety risk. Medical and manufacturer reports have primarily focused on larger electrosurgical equipment used in hospitals and their effect on IEDs. Our purpose was to provide a clinical review of commonly encountered IEDs, their clinical indications, potential interference with officebased electrosurgical equipment, and recommendations to prevent complications and injury.

\section{IEDs: Clinical Indications}

\section{Cardiac Pacemakers}

Although pacemaker technology has advanced since the first one was implanted in $1958,{ }^{3}$ common indications are still symptomatic bradyarrhythmias, such as sinus node dysfunction and heart block. A pacemaker consists of a battery-powered generator connected to electronic pacing wires inserted intravenously to the right-side chambers of the heart. ${ }^{4}$ The earliest devices were asynchronous devices, pacing the heart at a fixed rate regardless of the patient's underlying rhythm and sometimes causing symptomatic hemodynamic consequences. The latest models are demand pacemakers that sense the heart's intrinsic rhythm and inhibit pacing in the same chamber or trigger pacing in another cardiac chamber. $^{5,6}$ These devices also have sophisticated timing features to optimize heart rates and even treat some atrial arrhythmias.

\section{Implantable Cardioverter Defibrillators}

Implantable cardioverter defibrillators (ICDs) have had a profound effect on patients with ventricular tachyarrhythmias and those at risk for sudden cardiac death. ${ }^{7}$ ICDs are programmed to treat lifethreatening ventricular arrhythmias with rapid overdrive pacing or an internal defibrillator shock. These patients most commonly have severe acquired, inherited, or idiopathic forms of cardiomyopathy. Indications continue to expand to include asymptomatic patients with low ejection fractions at risk of sudden death. Similar to pacemakers, these devices

\footnotetext{
${ }^{*}$ Department of Dermatology, ${ }^{\dagger}$ Division of Cardiovascular Medicine, Department of Internal Medicine, ${ }^{\S}$ Department of Otolaryngology, and Division of Plastic Surgery, Department of Surgery, University of Michigan Medical School and Comprehensive Cancer Center, Ann Arbor, Michigan; ${ }^{\ddagger}$ Canton Dermatology PC, Canton, Michigan
} 


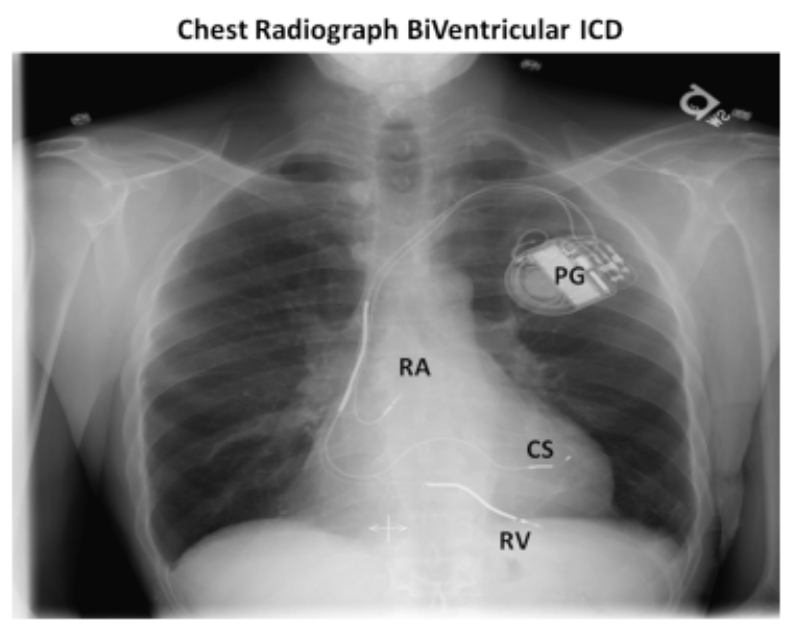

Figure 1. Chest radiograph of a patient with an implanted cardioverter defibrillator. PG, pulse generator; RA, pacemaker lead in the right atrium; CS, pacemaker lead in the coronary sinus; RV, defibrillator lead in the right ventricle.

consist of a pulse generator implanted subcutaneously in an infraclavicular pocket and connected to one or more leads for defibrillation and pacing ${ }^{5}$ (Figure 1). The generator or battery component of ICDs and pacemakers requires replacement approximately every 5 years. Some patients with ICDs or pacemakers receive biventricular pacing or cardiac resynchronization therapy. These devices have the additional capability of pacing the right and the left ventricle through a pacing lead placed through the coronary sinus. These are used to improve the survival and symptoms of patients with moderate to severe congestive heart failure, left ventricular dysfunction (left ventricular ejection fraction $<0.35$ ) and an abnormally wide QRS on electrocardiogram who remain symptomatic despite optimal heart failure drug therapy. ${ }^{6,8}$

\section{Cochlear Implants}

A cochlear implant is an option for individuals with hearing loss when hearing aids provide limited or no benefit. A cochlear implant system consists of two main parts. The first is the internal implant consisting of an electronics housing and the electrode array. The second is the external speech processor (worn behind the ear) containing a microphone and elec- tronics to process sound. Additional components include a cable, a transmitting coil, and a battery pack. ${ }^{9}$ An external sound processor captures sound and converts it into digital signals, which are then converted into electrical impulses by an internal implant and sent to an electrode array implanted into the cochlea. ${ }^{10}$ Electrical impulses from the cochlea stimulate the inferior root of the acoustic nerve. Neural discharges induce auditory sensations at the level of the brain cortex, restoring partial hearing. ${ }^{11}$ Candidates include children aged 12 months and older who have profound bilateral sensorineural hearing loss and adults with severe to profound bilateral sensorineural hearing loss who receive little or no benefit from traditional hearing aids. $^{9}$

\section{Deep Brain Stimulators}

Deep brain stimulation is a targeted neurosurgical intervention that enables an implanted pacemaker to electrically stimulate structures deep within the brain. ${ }^{1}$ The system was approved by the FDA in $1997^{12}$ for use in patients with drug-refractory essential tremor or Parkinsonian tremor that constitutes a significant functional disability. Deep brain stimulation is also indicated as an aid in the management of chronic, intractable primary dystonia, including generalized and segmental dystonia, hemidystonia, and cervical dystonia (torticollis) in individuals aged 7 and older. ${ }^{13}$ The device consists of a lead composed of a thin coiled wire with four electrodes stereotactically implanted in various sites of the brain. The lead is connected to a thin, insulated, coiled wire tunneled subcutaneously from the head and neck to the chest, where it attaches to a pulse generator implanted in a subcutaneous pocket in the anterior chest wall. Patients have the ability to turn the device on or off and switch between settings using an external magnet. ${ }^{4,14}$ Targets for stimulation include the internal globus pallidus, subthalamic nucleus, and thalamus. ${ }^{1,4,13}$ Implanting a device at these sites allows for individualized programming of stimulator impulse amplitude and frequency for optimal symptom management. ${ }^{15}$ 


\section{Vagal Nerve Stimulators}

The FDA approved the use of vagal nerve stimulators for the management of medically refractory epilepsy in 1997. ${ }^{1,12}$ Since then, indications for the use of these devices have expanded to include depression. ${ }^{16,17}$ Additional indications under investigation include obesity, Alzheimer's disease, chronic pain syndromes, ${ }^{18}$ anxiety, bulimia, and migraine headaches. ${ }^{16}$ Candidates for vagal nerve stimulator implantation include adults and adolescents aged 12 and older with partial-onset seizures refractory to medications and patients aged 18 and older with chronic or recurrent major depression without adequate response to at least four antidepressant treatments. ${ }^{17}$

Vagal nerve stimulators consist of a constant-current pulse generator, a single subcutaneously placed lead wire, and a silicone rubber-imbedded platinum electrode wrapped around the left vagus nerve. ${ }^{18}$ The implantable, multiprogrammable pulse generator is housed in a hermetically sealed titanium case and is powered by a single battery. The lead transmits electrical signals from the pulse generator to the vagus nerve. ${ }^{19}$ The left vagus nerve is preferred over the right because there are more cardiac efferent fibers from the right vagus nerve, stimulation of which may result in more-frequent adverse cardiac effects. Programming of the device occurs by placing an external programming wand on the skin overlying the generator. Radio frequency signals communicate between the generator and software on a standard personal computer or personal digital assistant. ${ }^{18}$

\section{Sacral Nerve Stimulators}

Sacral nerve stimulation was developed to treat incontinence in patients with severe neurologic disease. ${ }^{1,12}$ In 1997, the device was approved for urge incontinence and in 1999 for nonobstructive urinary retention and significant symptoms of urgency frequency refractory to medications and behavioral therapy. ${ }^{13}$ Other indications include interstitial cystitis, pelvic pain, and fecal incontinence. The device consists of a generator implanted subcutaneously in the hip area with a lead wire (containing four platinum electrodes) surgically inserted into the sacral foramen at $\mathrm{S} 3$ on one side adjacent to the sacral nerve. ${ }^{1}$ Patients have the ability to turn the device on and off using an external control unit. ${ }^{12}$

\section{Phrenic Nerve Stimulators}

A phrenic nerve stimulator provides ventilatory support for patients with chronic respiratory insufficiency whose diaphragm, lungs, and phrenic nerves have residual function. ${ }^{20}$ Candidates for diaphragm pacing include, but are not limited to, patients who require chronic ventilatory support because of central alveolar hypoventilation, decreased day or night ventilatory drive (sleep apnea, Ondine's curse), or brain stem or spinal cord injury, or disease. ${ }^{21}$ The pacing system electrically stimulates the phrenic nerve to cause rhythmic contraction of the diaphragm. ${ }^{1}$ It consists of electrodes sutured to each phrenic nerve, a radio receiver implanted in a subcutaneous pocket, and an external battery-powered transmitter and antenna assembly. The external transmitter and antenna send energy and stimulus information to the passive receiver implant, which transmits electrical impulses to the phrenic nerve by the electrode. The diaphragm muscle contracts, producing the inhalation phase of breathing. The transmitter then stops generating signals, which allows the diaphragm to relax, and exhalation occurs. This continuous cycle produces a normal breathing pattern. ${ }^{21}$

\section{Spinal Cord Stimulators}

Spinal cord stimulators aid in the management of chronic, intractable pain of the trunk and limbs, including unilateral or bilateral pain refractory to conservative and surgical interventions often associated with conditions such as chronic low back pain syndrome, radiculopathy, postsurgical pain, degenerative disk disease or herniated disk, peripheral causalgia, epidural fibrosis, arachnoiditis, and complex regional pain syndrome. Spinal cord stimulation involves the delivery of mild electrical signals to the epidural space. The perception of paresthesia in the area where pain is felt "masks" pain signals. ${ }^{13}$ Leads are typically implanted under 
fluoroscopic guidance into the epidural space adjacent to the lower aspect of the spinal cord between levels T9 and L1 or open placement in the cervical region. A generator is usually implanted anteriorly using a tunneled approach between the skin and fascial layers. ${ }^{12}$ Electric current produced by the pulse generator is transferred to the lead generating paresthesia. Patients can use an external programmer to adjust intensity and location of stimulation within clinician-set parameters to meet pain management needs. ${ }^{13}$

\section{Gastric Pacemaker}

Gastric electrical stimulation was advocated as a possible treatment for gastric motor dysfunction as early as $1963 .^{22}$ In 2000, the FDA approved the device for human use for the treatment of chronic intractable nausea and vomiting secondary to gastroparesis of diabetic or idiopathic etiology. ${ }^{1,13,22}$ In addition, it is under investigation as an adjuvant therapy to blunt appetite and reduce food intake in morbidly obese people. ${ }^{22,23}$ The device consists of a small battery-powered neurostimulator implanted in a subcutaneous pocket of the lower abdominal region connected to two intramuscular leads implanted in the muscle wall of the antrum of the stomach. A handheld, external programmer noninvasively adjusts therapy specifically for each patient, assesses neurostimulator battery status, and can perform troubleshooting as needed. ${ }^{13}$ All devices have a phasic stimulation pattern, with an initial on time during which a pulse or series of pulse bursts are administered, followed by an off period without current delivery. On times range from 0.1 to $4 \mathrm{sec}-$ onds and off times from 1 to 5 seconds and are set based on treatment protocols for gastroparesis and obesity. Parameters such as pulse frequency and duration can be modified to elicit specific motor and symptom responses. ${ }^{23}$ The exact mechanism underlying gastric pacing for gastroparesis is not well understood. It is thought that gastric pacing generates high-frequency stimuli, which enhance motility and facilitate emptying. ${ }^{1,12}$ Gastric stimulation for obesity is thought to augment antral contractions, enhancing satiety elicited by postprandial gastric distention. $^{23}$

\section{Bone Stimulator}

Bone has an inherent bioelectrical property generated by cellular metabolism. Being rich in calcium phosphate crystals, it also exhibits piezoelectric properties when subjected to mechanical stress. Both are thought to be important in modifying a variety of processes in the callus and remodeling phases of fracture healing. An understanding of these properties coupled with an accumulating body of evidence on the positive effect of electric stimulation on osteoprogenitor cell production has led to the application of electrostimulation to accelerate bone healing. ${ }^{24}$ Bone stimulators are used as an adjunct to planned surgery or when adherence is of concern in patients with nonunions. They may also be used as an adjunct to internal and external fixation and autograft. ${ }^{25}$ Invasive and noninvasive bone stimulators are available. Noninvasive devices use an electromagnetic field to stimulate growth and consist of a generator and electrodes attached to the surface of the skin. Invasive devices use direct current and involve the implantation of electrodes at a desired site for bone growth. Negative electrodes are placed at the site of bone repair, and positive electrodes are placed in the soft tissue. A generator is implanted subcutaneously nearby or in an intramuscular plane. ${ }^{1}$ A third, partially invasive method involves the implantation of one lead into the fracture site and of a second lead on the skin. The generator is incorporated into a cast. ${ }^{12}$

\section{Electrosurgical Equipment}

\section{Heat Electrocautery, Nongrounded Monopolar, Grounded Monopolar, and Bipolar Devices}

Most outpatient dermatologic surgery procedures employ some form of external energy source for hemostasis. There are two general methods to obtain hemostasis: electrocautery and electrosurgery. Each has an electrical power source, but their interference with IEDs varies, each carrying a different risk to the 
patient, making knowledge of the IED and equipment selection important. Even though the risk for EMI is low with many IEDs - depending on the procedure and method of hemostasis - the potential for harm can be reduced with equipment selection. Thus, preoperative evaluation for the office-based dermasurgeon in patients with IEDs includes the challenge of balancing adequate hemostasis with the risk of EMI.

Heat electrocautery does not use electric current ${ }^{5,26}$; it converts electrical energy at high resistance into thermal energy, heating a metal tip, which produces hemostasis when held in direct contact with tissue. ${ }^{4}$ Because no electric current passes to the patient, heat electrocautery is the safest instrument for hemostasis in patients with IEDs for office-based procedures when the risk of EMI is high. Risk of thermal damage to an implant from direct heat is minimal and only of concern when using the device directly over the IED. ${ }^{4,5}$

Broadly, electrosurgery is a high-frequency alternating electrical current through an unheated electrode. $^{26}$ Two methods of current can be delivered: monopolar and bipolar. In monopolar electrosurgery, current delivered to the surgical site is through one electrode. High-powered electrosurgical units, found in hospital operating rooms, are typically monopolar units, generating current that travels from the electrode instrument to the surgical site through the patient's body to a grounding pad (return electrode) and back to the electrosurgical unit to complete a circuit. In contrast, office-based monopolar electrosurgical units (e.g., Hyfrecator, Conmed Corp, Utica, NY) are low powered, making a grounding pad optional. When a grounding pad is absent, current disperses throughout the body. This is a relatively safe means of obtaining hemostasis in a majority of patients and is commonly used for routine office-based dermatologic procedures, but because current flows beyond the surgical site, the use of a low-powered unit without a grounding pad has the potential to cause interference in patients with an IED. In bipolar electrosurgery, current travels through a two-electrode instrument from one electrode, through tissue, to a second adjacent elec-
Electrosurgical Techniques

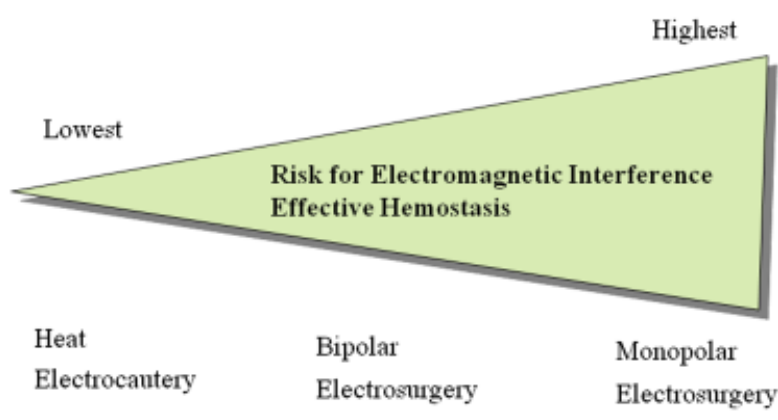

Figure 2. Electromagnetic interference risk and effective hemostatic capability of different electrosurgical techniques.

trode, completing an electrical circuit. Because current through bipolar instruments is concentrated across the tips, a grounding pad is not required, and electrical energy capable of interfering with an IED is minimized $^{4}$ (Figure 2).

\section{General Considerations}

Information regarding the presence or absence of an IED should be obtained from each patient before planned procedures such as Mohs, postprocedure reconstruction, electrodesiccation and curettage, and standard excisions and biopsies in which electrosurgery may be used. For unplanned procedures, verbal confirmation of the presence or absence of an IED should be obtained from each patient before performing electrosurgery. Once identified, preoperative evaluation should focus on the type of device, its medical indication, type and extent of procedure, and amount of bleeding expected. Type and location of IED, date of implantation and last interrogation, programmability, symptoms when device is turned off, and need for postoperative interrogation are also important. ${ }^{1}$ The type of electrosurgery should be planned based on the anticipated surgical procedure. Heat electrocautery or bipolar instead of monopolar electrosurgery is favored for unmonitored procedures, because these are generally safer in patients with IEDs. ${ }^{27}$ For procedures of higher complexity, physicians should also consult the patient's primary 
device specialty physician and consider consulting an industry representative or device specialist for additional intraoperative safety measures.

\section{Electrosurgery and Implanted Cardiac Devices}

Electrosurgery-associated EMI has resulted in a small number of inappropriate episodes of device malfunction, including suspension of arrhythmia detection and delivery of a defibrillatory shock in patients with ICDs. ${ }^{6,7,28-32}$ More-recent reports suggest that modern cardiac pacemakers have greater immunity from EMI than earlier devices. ${ }^{2}$ Despite these advances, the sensing circuitry does not filter out some types of EMI, which may be erroneously interpreted as a rapid heart rate. For instance, if EMI is persistent, an ICD can misinterpret it as tachyarrhythmia, resulting in inappropriate delivery of shock therapy, ${ }^{33}$ but newer ICDs have incorporated filters to detect EMI, making this risk very low. ${ }^{31,34}$ Surgeons should not fear transmission of the shock to themselves or others in contact with the patient because most of the electric force dissipates before reaching the patient's skin. ${ }^{35,36}$

The earliest nondemand pacemakers lacked the capability to sense electromagnetic signals and are rarely encountered today, ${ }^{5}$ but virtually all modern pacemakers and ICDs are demand devices and could be affected by EMI. The most commonly encountered response of demand pacemakers to EMI is oversensing, resulting in pacing inhibition. ${ }^{28,37-39}$ Other responses include inappropriately triggered pacing $^{5,7}$ or pacemaker reprogramming to a default mode. ${ }^{6,31,37}$ Although it is not known how frequently this occurs, large amounts of electrical energy during electrosurgery could interfere with a pacemaker's pulse generator, damaging the internal device circuitry. There are rare reports of energy transfer to the myocardium through the pacer lead, resulting in thermal damage at the lead-tissue interface. ${ }^{26,29,31,32,37,40}$ Because the energy levels encountered in most office dermasurgical suites is not commonly associated with these reported inter- ferences, the exact frequency of EMI with IEDs in an office dermasurgery practice is not known.

Attempts to lessen the effects of EMI on pacemakers have included metallic shielding to protect against high-frequency EMI; noise sampling periods converting pacemakers to a fixed rate if continuing EMI is sensed; bipolar leads, which have improved the rejection of noise over the original unipolar lead ${ }^{41}$; and "bandpass filters" filtering nonphysiologic signals outside the narrow range of cardiac depolarization frequencies. ${ }^{5,31}$ These improvements have significantly reduced the frequency of EMI events with cardiac devices. ${ }^{42}$

Pacemaker-dependent patients are a special subgroup to analyze before electrosurgery. Patients who have no underlying rhythm other than the paced rhythm are considered pacemaker dependent and should be identified, because prolonged inhibition of a paced rhythm could lead to symptoms. Roughly $5 \%$ to $10 \%$ of patients with implantable cardiac pacemakers are dependent on the device for maintaining adequate heart rhythm. ${ }^{34,42,43}$ Only the cardiologist or device representative may know this status. Asynchronous pacing is thought to minimize the potential for reversion or inhibition due to oversensing. Although this preoperative programming change is standard for pacemaker-dependent patients, complications have been reported. One report described EMI from a monopolar device causing battery depletion and eventual "voltage control oscillator lock-out," with subsequent pacemaker failure in a 15 -year-old patient after cardiac surgery. ${ }^{2}$ In a second report, sudden irreversible output loss after the use of bipolar electrosurgery in short bursts at a site more than 12 inches from the pulse generator occurred. ${ }^{44}$ The generator was subsequently explanted and found to be functioning in the "reset" mode several weeks later. Initial pacemaker failure was postulated to be due to a "voltage control oscillator lock-out," but it was unknown whether the pacemaker was functioning in the "reset" mode because of exposure to cold temperatures during transport to the manufacturer or to further use of an 
electrosurgical device during explantation. These rare but dangerous complications highlight the need even for the office-based dermasurgeon to use electrosurgical equipment with the least amount of risk for EMI in patients with cardiac implants. For this reason, pacemakers and ICDs of known pacemaker-dependent patients at the University of Michigan are reprogrammed to an asynchronous mode before most surgeries requiring electrosurgery with continuous intraoperative monitoring. ${ }^{34,38}$

\section{Large Modern Series of EMI in Cardiac Implants}

A cross-sectional prospective study of 92 consecutive patients who underwent noncardiac surgical or endoscopic procedures found infrequent cardiac device malfunction related to EMI. ${ }^{42}$ Patients underwent preand postoperative interrogation of their pacemakers or ICDs. Rare inappropriate sensing by devices was reported with monopolar electrosurgical application in close proximity to a cardiac implant. All devices withstood periprocedural EMI exposure without malfunction or changes in programming. Only minor changes in lead parameters were found, along with three devices resetting briefly to an atrial arrhythmia response mode (mode switching) and two pacemakers inappropriately sensing ventricular noise with electrosurgical application within $8 \mathrm{~cm}$ of the pacemaker generator. These changes had no immediate clinical detriment.

A retrospective review of 173 patients with a pacemaker and 13 with an ICD who underwent Mohs or dermatologic excisional surgery involving electrosurgery was performed at the Mayo Clinic. ${ }^{45}$ All patients had preoperative cardiology evaluation or device information reviewed by a staff member who deemed preoperative cardiology consultation unnecessary. Twelve of the 13 patients with an ICD underwent preoperative device deactivation with continuous intraoperative monitoring until postoperative device reactivation. The remaining patient with an ICD was advised against electrosurgery due to pacemaker dependence and therefore received heat electrocautery for hemostasis. Patients received bipolar electrosurgery using short bursts of low- voltage electrical activity. No operation-related device malfunctions or complications were identified after postoperative interrogation. ${ }^{45}$

\section{Academic Medical Center Experience}

Despite the favorable outcome reported above, the risk of EMI is not zero. Until a consensus statement from large medical societies (such as the Heart Rhythm Society) is published, our institution has taken a cautious approach, recognizing that these recommendations may not always be agreed upon or practical for all office-based procedures. Specific EMI complications from dermasurgery have not been reported in a highlevel evidence fashion, and until studies on the relative safety of one method of hemostasis over another are reported, some simple precautions are made.

Because bipolar electrosurgery and heat electrocautery have excellent safety profiles, these modalities are considered optimal. ${ }^{27}$ Over the past three decades in our institution, we have not observed any known EMI with IEDs using this approach. When the risk of bleeding is high, such as with larger lesions, in vascular locations, or in patients taking anticoagulants, precautions are taken. Until data are reported or national guidelines are published, all ICD patients at our institution are continuously monitored during electrosurgery, with emergency pacing and defibrillation equipment readily available. If the implanted device malfunctions, a longer time between bursts is implemented to attempt to minimize hemodynamic effects. The grounding pad is placed so that current flowing between the surgical site and the grounding pad will not intersect the pacing system. Device function is evaluated postoperatively to identify setting changes that may have occurred intraoperatively (Medtronic Technical Services, personal communication, September 2009). 2,34

Many industry representatives and cardiologists do not believe that all devices need to be routinely evaluated immediately after surgery because there have been no reported instances of EMI-induced permanent reprogramming, and it may not be feasible in all practice settings to obtain an 


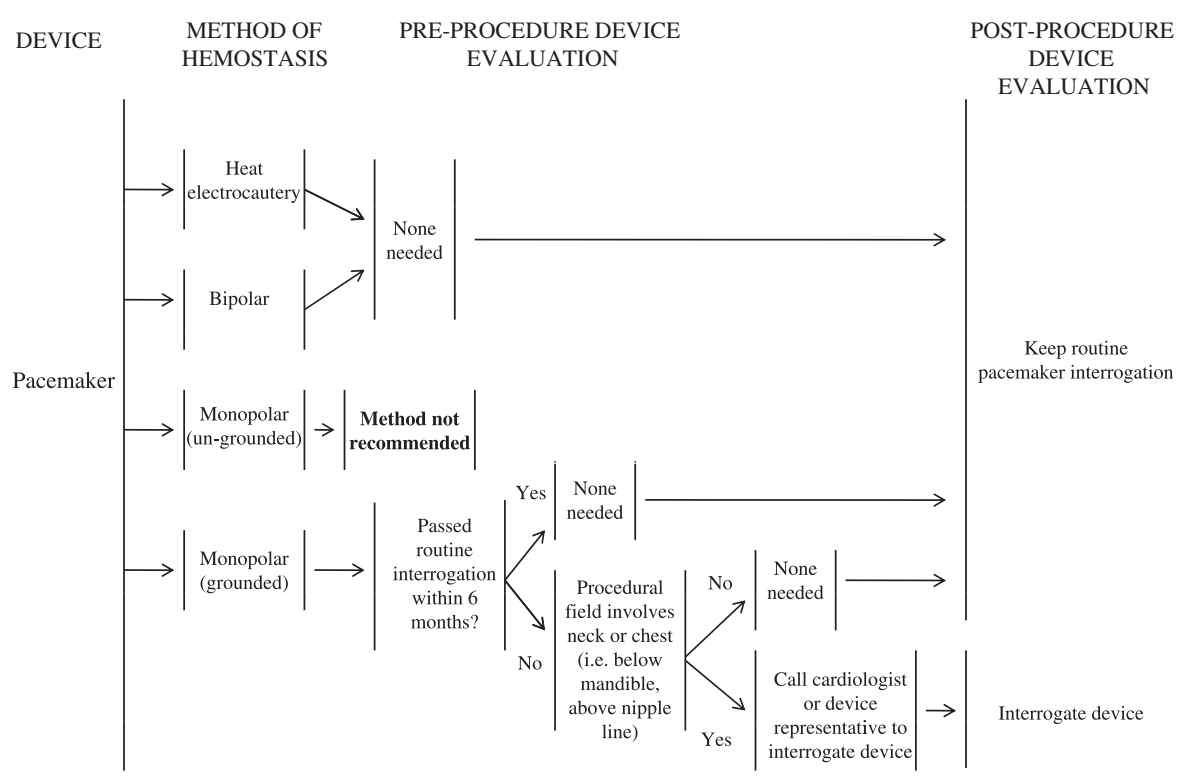

Figure 3. University of Michigan periprocedural management for a pacemaker patient for planned, unmonitored officebased procedures.

evaluation. Should an office-based dermasurgery proceed because of practical issues, then electrosurgery should not be performed within 6 inches $(15 \mathrm{~cm})$ of a cardiac device or lead system without prior consultation. Minimum power settings and short, intermittent, irregular bursts $(<1$-second duration) should be used, but interrogation should be performed if one suspects any change of device function or settings have occurred. ${ }^{6}$ Refer to Figures 3 and 4 for University of Michigan periprocedural pacemaker and ICD management algorithms.

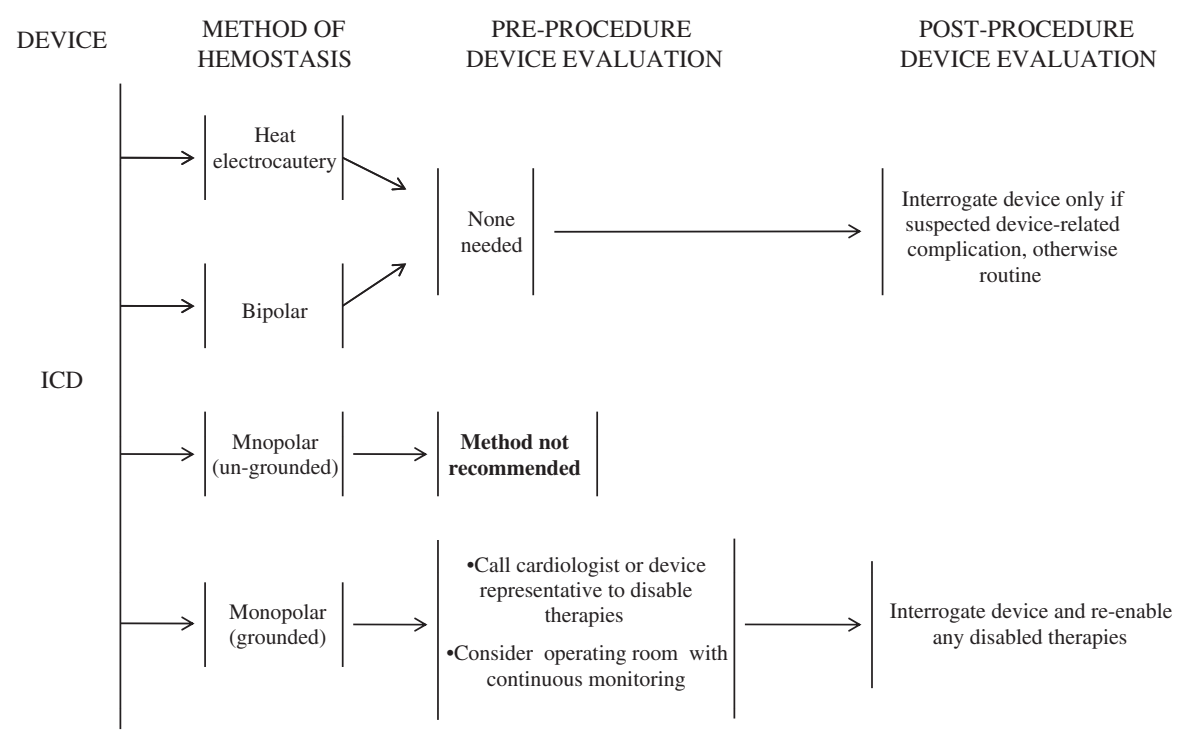

Figure 4. University of Michigan periprocedural management of a patient with an implantable cardioverter defibrillator (ICD) for planned, unmonitored office-based procedures. 


\section{Electrosurgery and Implanted Noncardiac Devices}

\section{Cochlear Implants}

EMI has the potential to cause irreparable damage to cochlear devices, necessitating a surgical procedure for replacement. Electrosurgical instruments are capable of producing voltages of such magnitude that a direct coupling can exist between the electrosurgical tip and a cochlear implant electrode. In addition to implant damage, sufficient electrical energy could necrotize vital cells of the basilar membrane, making re-implantation futile. ${ }^{11}$ The manufacturer, Med-EL Corporation, states that monopolar use is strictly prohibited on any patient with cochlear devices and supports the use of bipolar electrosurgery as long as it is kept at least $3 \mathrm{~cm}$ from the implant package and electrodes (S. Greene, personal communication, September 2009). Further recommendations from Med-EL include removing all external equipment before surgical procedures. Active and return electrosurgical electrodes should not be in the head or neck region. In addition, precautions should be taken to prevent flow of electric current over the head and neck region of the patient. This includes avoiding conductive underlay or conductive blanket use and current flow across the head from monitor cables (S. Greene, personal communication, September 2009).

\section{Deep Brain Stimulators}

Data on the effects of electrosurgery on deep brain stimulators is limited, but the absence of sound data does not permit excluding it as a potential health concern. ${ }^{15}$ Although EMI does not appear to present an immediate safety concern in patients with deep brain stimulators, device malfunction with resulting temporary interruption in therapeutic benefit may occur. ${ }^{15}$ We found only two case reports of Mohs surgery performed on patients with deep brain stimulators. In one report, hemostasis was obtained using heat electrocautery without discomfort or complications. ${ }^{4}$ The other reported using monopolar electrosurgery without a grounding pad for hemostasis. The patient experienced immediate lancinating "electric shock" sensations down his left arm and leg. Monopolar electrosurgery was discontinued, and heat electrocautery was used for the remainder of the procedure, without discomfort. Postoperative assessment did not reveal any changes in device parameters. The patient required additional dermatologic procedures in which two different electrosurgical methods were used. The patient once again experienced electric shock sensations when a monopolar pencil was used to achieve hemostasis of a left preauricular lesion with grounding pad on the right posterior calf. Bipolar forceps were used in a subsequent procedure without discomfort. In a third procedure, a monopolar pencil was once again used for a lesion on the left cheek. The grounding pad was positioned on the left trapezius, so that it was closer to the electrical output yet away from the device. The patient did not report any discomfort with this arrangement. ${ }^{14}$

\section{Other Devices}

Manufacturers warn that EMI from electrosurgical application can result in damage to devices and their components, as well as temporary changes in neurostimulator output, reprogramming, and failure in deep brain stimulators, vagal nerve stimulators, sacral nerve stimulators, spinal cord stimulators, and gastric pacemakers. ${ }^{13,17,46-50}$ In addition, currents induced in the lead portion of the neurostimulation system can be hazardous or cause further injury. ${ }^{48-50}$ To minimize these risks, deep brain stimulators, sacral nerve stimulators, spinal cord stimulators, and gastric pacemakers may be turned off before electrosurgical application. ${ }^{14,48-50}$ Deactivation of a vagal nerve stimulator is not necessary ${ }^{18}$ and should not be performed in patients with bone stimulators (K. Edwards, personal communication, October 2009).

Heat electrocautery should be used for these devices when appropriate, because it poses no risk to the patient as long as it is used away from the device. When performing electrosurgery, bipolar is recommended away from the stimulator or lead. ${ }^{46-50}$ If monopolar is necessary, a low-voltage mode should 
be used on the lowest possible power setting, keeping the grounding pad as far from the neurostimulator, extension, and lead as possible. ${ }^{46-50}$ A manufacturer website states that monopolar electrosurgery should not be used on patients with spinal cord stimulators. ${ }^{8}$ Care should be taken to avoid electrode placement that puts a pulse generator or lead in the direct path of current flow or within the area of the body being treated. ${ }^{19,49}$ In addition, cables connecting a lead or extension to an external neurostimulator should be disconnected in sacral nerve stimulators and spinal cord stimulators. ${ }^{48,50}$ Finally, postoperative evaluation of a neurostimulator should be performed for proper functioning. ${ }^{19,48-50}$

Our literature search did not reveal any specific recommendations pertaining to electrosurgery in patients with phrenic nerve stimulators and bone stimulators. In addition, specific warnings and recommendations were not found on manufacturer websites. Personal communication with manufacturer representatives revealed a recommendation of bipolar electrosurgery on patients with phrenic nerve stimulators (K. Aron, personal communication, September 2009) and warnings that electrosurgical devices may cause bone stimulators to be "shorted out" or rendered ineffective and could result in local tissue damage (K. Edwards, personal communication, October 2009).

\section{Conclusion}

Technological advances have prompted the implantation of a variety of electronic devices for a myriad of cardiac and neurological disorders that cannot be managed using medication alone. Encountering patients with an IED who present for an office-based procedure is becoming increasingly common. Potential EMI is a concern for physicians performing procedures using electrosurgery. Understanding potential complications when performing electrosurgery in this group of patients is vital for patient safety. Device-specific perioperative guidelines are needed to ensure safety when performing electrosurgery in patients with modern IEDs. The perioperative management of pacemakers and ICDs is a major focus for organizations such as the Heart Rhythm Society, which is preparing a document to address this topic. This will also provide a stimulus for clinical research to advance our understanding and improve clinical care.

\section{References}

1. Venkatraghavan L, Chinnapa V, Peng P, Brull R. Non-cardiac implantable electrical devices: brief review and implications for anesthesiologists. Can J Anesth 2009;56:320-6.

2. Mangar D, Atlas GM, Kane PB. Electrocautery-induced pacemaker malfunction during surgery. Can J Anesth 1991;38:616-8.

3. Mond HG, Sloman JG, Edwards RH. The first pacemaker. Pacing Clin Electrophysiol 1982;5:278-82.

4. Martinelli PT, Schulze KE, Nelson BR. Mohs micrographic surgery in a patient with a deep brain stimulator: a review of the literature on implantable electrical devices. Dermatol Surg 2004;30:1021-30.

5. Yu SS, Tope WD, Grekin RC. Cardiac devices and electromagnetic interference revisited: new radiofrequency technologies and implications for dermatologic surgery. Dermatol Surg 2005;31:932-40.

6. Stone ME, Apinis A. Current perioperative management of the patient with a cardiac rhythm management device. Semin Cardiothorac Vasc Anesth 2009;13:31-43.

7. Occhetta E, Bortnik M, Magnani A, Francalacci G, et al. Inappropriate implantable cardioverter-defibrillator discharges unrelated to supraventricular tachyarrhythmias. Europace 2006;8:863-9.

8. Boston Scientific Products [on-line]. Available from: http:// www.bostonscientific.com/Device.bsci/,/method/DevHome/ navRelId/1000.1003/seo.serve Accessed August 26, 2009.

9. Med-EL Corporation [on-line]. Available from: http://www. medel.com/US/ Accessed August 26, 2009.

10. Cochlear Limited: Introducing Nuclear 5 [on-line]. Available from: http://products.cochlearamericas.com/cochlear-implants/ our-cochlear-implant-products. Accessed August 26, 2009.

11. Tognola G, Parazzini M, Sibella F, Paglialonga A, et al. Electromagnetic interference and cochlear implants. Ann Ist Super Sanita 2007;43:241-7.

12. Levin G, Ortiz AO, Katz DS. Noncardiac implantable pacemakers and stimulators: current role and radiographic appearance. Am J Roentgenol 2007;188:984-91.

13. Medtronic for Healthcare Professionals [on-line]. Available from: http://www.medtronic.com/for-physicians/index.htm. Accessed August 26, 2009.

14. Weaver J, Kim SJ, Torres A. Cutaneous electrosurgery in a patient with a deep brain stimulator. Dermatol Surg 1999;25:415-7.

15. Dustin K. Evaluation of electromagnetic incompatibility concerns for deep brain stimulators. J Neurosci Nurs 2008;40:299-303.

16. Cyberonics, Inc. [on-line]. Available from: http://us.cyberonics. com/en. Accessed June 10, 2010.

17. Brief Summary of Safety Information for the VNS Therapy System. Available from: http://www.vnstherapy.com/epilepsy/hcp/ indications_for_use.aspx. Accessed August 28, 2009. 
18. Hatton KW, McLarney JT, Pittman T, Fahy BG. Vagal nerve stimulation: overview and implications for anesthesiologists. Anesth Analg 2006;103:1241-9.

19. Physician's Manual VNS Therapy Demipulse Model 103 Generator and VNS Therapy Demipulse Duo Model 104 Generator [online]. Available from: http://www.vnstherapy.com/manuals/. Accessed October 9, 2009.

20. Avery Breathing Pacemakers [on-line]. Available from: http:// www.averylabs.com/index.html. Accessed August 27, 2009.

21. Instruction Manual for the Mark IV Breathing Pacemaker System. Commack, NY: Avery Biomedical Devices. Inc.; 2009.

22. Zhang J, Chen JD. Systematic review: applications and future of gastric electrical stimulation. Aliment Pharmacol Ther 2006; 24:991-1002.

23. Hasler WL. Methods of gastric electrical stimulation and pacing: a review of their benefits and mechanisms of action in gastroparesis and obesity. Neurogastroenterol Motil 2009;21:229-43.

24. Kesani AK, Gandhi A, Lin SS. Electrical bone stimulation devices in foot and ankle surgery: types of devices, scientific basis and clinical indications for their use. Foot Ankle Int 2006;16:148-56.

25. OsteoGen Bone Growth Stimulator [on-line]. Available from: http://www.biomet.com/trauma/products.cfm? pdid=4\& majcid= 47\& prodid=268. Accessed August 28, 2009.

26. LeVasseur JG, Kennard CD, Finley EM, Muse RK, et al. Dermatologic electrosurgery in patients with implantable cardioverter-defibrillators and pacemakers. Dermatol Surg 1998;24:233-40.

27. El-Gamal HM, Dufresne RG, Saddler K. Electrosurgery, pacemakers and ICDs: a survey of precautions and complications experienced by cutaneous surgeons. Dermatol Surg 2001;27:385-90.

28. Dyrda K, Khairy P. Implantable rhythm devices and electromagnetic interference: myth or reality? Expert Rev Cardiovasc Ther 2008;6:823-32.

29. Sweesy MW. Understanding electromagnetic interference. Heart Rhythm 2004;4:523-4.

30. Kolb C, Zrenner B, Schmitt C. Incidence of electromagnetic interference in implantable cardioverter defibrillators. Pacing Clin Electrophysiol 2001;24(4 Pt 1):465-8.

31. Madigan JD, Choudhri AF, Chen J, Spotnitz HM, et al. Surgical management of the patient with an implanted cardiac device. Ann Surg 1999;230:639-47.

32. Yerra L, Reddy PC. Effects of electromagnetic interference on implanted cardiac devices and their management. Cardiol Rev 2007;15:304-9.

33. Magnet Use for Suspending Medtronic ICD Detection [on-line]. Available from: http://cacgas.com/uploads/Medtronic__Magnet_ use_and_ICD.pdf. Accessed September 8, 2009.

34. Dawes JC, Mahabir RC, Hillier K, Cassidy M, et al. Electrosurgery in patients with pacemakers/implanted cardioverter defibrillators. Ann Plast Surg 2006;57:33-6.

35. Wolbrette DL, Naccarelli GV. Management of implantable cardioverter defibrillator patients: role of predischarge electrophysiologic testing and proper patient instruction before hospital discharge. Curr Opin Cardiol 2001;16:72-5.

36. Hazelton GA, Sears SF, Kirian K, Matchett M, et al. Coping with my partner's ICD and cardiac disease. Circulation 2009;120:e73-6.
37. Sweesy MW, Holland JL, Smith KW. Electromagnetic interference in cardiac rhythm management devices. AACN Clin Issues 2004;15:391-403.

38. Niehaus M, Tebbenjohanns J. Electromagnetic interference in patients with implanted pacemakers or cardioveter-defibrillators. Heart 2001;86:246-8.

39. Heller LI. Surgical electrocautery and the runaway pacemaker syndrome. Pacing Clin Electrophysiol 1990;13:1084-5.

40. Snow JS, Kalenderian D, Colasacco JA, Jadonath RL, et al. Implanted devices and electromagnetic interference: case presentations and review. J Invasive Cardiol 1995;7:25-32.

41. Wilson S, Neustein SN, Camunas J. Rapid ventricular pacing due to electrocautery: a case report and review. Mt Sinai J Med 2006;73:880-3.

42. Cheng A, Nazarian S, Spragg D, Bilchick K, et al. Effects of surgical and endoscopic electrocautery on modern-day permanent pacemaker and implantable cardioverter-defibrillator systems. Pacing Clin Electrophysiol 2008;31:344-50.

43. Nanthakumar K, Dorian P, Ham M, Lam P, et al. When pacemakers fail: an analysis of clinical presentation and risk in 120 patients with failed devices. Pacing Clin Electrophysiol 1998;21:87-93.

44. Peters RW, Gold MR. Reversible prolonged pacemaker failure due to electrocautery. J Interv Card Electrophysiol 1998;2:343-4.

45. Matzke TJ, Christenson LJ, Christenson SD, Atanashova N, et al. Pacemakers and implantable cardiac defibrillators in dermatologic surgery. Dermatol Surg 2006;32:1155-62.

46. Technical Manual for Kinetra Dual Program Neurostimulator for Deep Brain Stimulation [on-line]. Available from: http://profes sional.medtronic.com/products/kinetra-deep-brain-neurostimula tor/manuals-and-technical-resources/index.htm. Accessed September 10, 2009.

47. Physician and Hospital Staff Manual for Soletra Neurostimulator for Deep Brain Stimulation [on-line]. Available from: http://pro fessional.medtronic.com/products/soletra-deep-brain-neurostim ulator/manuals-and-technical-resources/index.htm. Accessed September 10, 2009.

48. InterStim Therapy Information for Prescribers [on-line]. Available from: http://professional.medtronic.com/products/interstimsacral-nerve-neurostimulator/manuals-and-technical-resources/ index.htm\#tab3. Accessed September 10, 2009.

49. Technical Manual for Enterra Therapy Gastric Electrical Stimulation System [on-line]. Available from: http://professional.med tronic.com/products/enterra-gastric-neurostimulator/manualsand-technical-resources/index.htm. Accessed September 10, 2009.

50. Medtronic Pain Therapy: Information for Prescribers [on-line]. Available from: http://professional.medtronic.com/products/ restoreadvanced-spinal-cord-neurostimulator/manuals-and-tech nical-resources/index.htm\#tab3. Accessed September 10, 2009.

Address correspondence and reprint requests to: Melissa A. Voutsalath, DO, University of Michigan Health System, 1147 Cancer and Geriatrics Center, 1500 E. Medical Center Drive, Ann Arbor, MI 48109-0918, or e-mail: mvoutsal@med.umich.edu 